\title{
Non-invasive score identifies ultrasonography- diagnosed non-alcoholic fatty liver disease and predicts mortality in the USA
}

\author{
Ching-Lung Cheung ${ }^{1,2,3,45^{*}}$, Karen SL Lam ${ }^{1,2,5}$, lan CK Wong ${ }^{3,5}$ and Bernard MY Cheung ${ }^{1,2,5^{*}}$
}

\begin{abstract}
Background: Several non-invasive prediction scores for non-alcoholic fatty liver disease (NAFLD) have been developed, but their performance has not been compared and validated in the same population, and whether these prediction scores can predict clinical outcomes remains unknown. In this study, we aimed to validate and compare the performance of four NAFLD prediction scores: fatty liver index, hepatic steatosis index, lipid accumulation product, and NAFLD liver fat score (LFS), and to evaluate the ability of the best NAFLD prediction score to predict mortality.

Methods: We analyzed data from the National Health and Nutrition Examination Survey conducted in 1988 to 1994, and subsequent follow-up data for mortality up to December 31, 2006. NAFLD was defined by ultrasonographic detection of hepatic steatosis in the absence of other known liver diseases.

Results: In a group of 5,184 participants, LFS consistently showed the highest area under the curve for predicting the presence of NAFLD. During a median follow-up of 14.7 years (range 0.1 to 18.2 years) and 83,830.5 person-years, participants in the high LFS group (LFS $\geq 1.257$ ) had a higher cardiovascular and liver-related mortality than participants in the low (LFS $\leq-1.413$; cardiovascular hazard ratio $(H R)=2.24,95 \% \mathrm{Cl} 1.03$ to 4.88 ; liver $\mathrm{HR}=31.25,95 \% \mathrm{Cl} 3.13$ to 333.33 ) or intermediate $(-1.413<\mathrm{LFS}<1.257$; cardiovascular HR=2.3, 95\% Cl 1.19 to 4.48 ; liver HR=30.3, 95\% Cl 4 to 250$)$ LFS groups in the fully adjusted model. Similar results were obtained when LFS was treated as a continuous variable.
\end{abstract}

Conclusions: LFS is the best non-invasive prediction score for NAFLD, and people with a high LFS score have an increased risk for cardiovascular and liver-related mortality.

Keywords: NAFLD, NAFLD liver fat score, Mortality

\section{Background}

Non-alcoholic fatty liver disease (NAFLD) represents a spectrum of progressive liver disease ranging from simple steatosis to non-alcoholic steatohepatitis (NASH), fibrosis, and cirrhosis, in the absence of excessive alcohol consumption. NAFLD is regarded as a hepatic manifestation of metabolic syndrome (MetS) [1], therefore the presence of NAFLD is not only strongly associated with liver-related mortality, but also with diseases related to the MetS, such as diabetes and cardiovascular diseases $[2,3]$. As NAFLD is highly prevalent and affects up to

\footnotetext{
* Correspondence: lung1212@hku.hk; mycheung@hku.hk

'Department of Medicine, Li Ka Shing Faculty of Medicine, The University of Hong Kong, Pokfulam, Hong Kong

${ }^{2}$ Research Centre of Heart, Brain, Hormone and Healthy Aging, Li Ka Shing Faculty of Medicine, The University of Hong Kong, Pokfulam, Hong Kong Full list of author information is available at the end of the article
}

$30 \%$ of the general adult population [4], screening for and diagnosing NAFLD has become an important issue in public health to prevent NAFLD-related complications and reduce healthcare costs.

Liver biopsy remains the "gold standard" for NAFLD diagnosis; however, this is an invasive technique making it impractical to be used widely. Ultrasonography is therefore the recommended first-line imaging technique in clinical practice, although it is known to have limited sensitivity [3]. Other non-invasive tools have been developed for diagnosing NAFLD, such as computed tomography and proton magnetic resonance spectroscopy $\left({ }^{1} \mathrm{H}\right.$-MRS). However, these tools are expensive and timeconsuming, and are not considered cost-effective for large-scale NAFLD screening. Recently, five biomarker- 
based non-invasive prediction scores of NAFLD have been developed: SteatoTest [5], fatty liver index (FLI) [6], NAFLD liver fat score (LFS) [7], lipid accumulation product (LAP) [8], and hepatic steatosis index (HSI) [9]. These scores are derived from simple clinical risk factors and biomarkers, and can therefore potentially be used for large-scale NAFLD screening. However, different definitions and techniques were used to define NAFLD in the original studies, and the performances of these scores have not been validated, evaluated, and compared directly in a large general population. In addition, whether these non-invasive scores of NAFLD can predict clinical outcome remains largely unknown.

In this study, we aimed to validate and evaluate the performance of these non-invasive prediction scores of NAFLD in predicting ultrasonography-diagnosed NAFLD in a representative general adult population in the USA (cross-sectional NAFLD prediction cohort), and to test if the marker can predict mortality in the general population (prospective mortality prediction cohort).

\section{Methods}

\section{Participant recruitment}

Data from the third National Health and Nutrition Examination Survey (NHANES III) were used [10]. NHANES III was conducted by the National Center for Health Statistics (NCHS) from 1988 to 1994, using a stratified multistage probability sample that represented the civilian non-institutionalized population in the USA. Participants gave written consent before participation, and ethics approval was obtained from the Human Subjects Committee of the US Department of Health and Human Services.

We studied people aged 20 to 74 years who participated in the NHANES III survey. Laboratory tests were carried out in a mobile examination center $(n=14,797)$ (see Additional file 1: Figure S1). Because all non-invasive score formulae require levels of biomarkers in fasting blood, we included participants with blood taken after fasting for at least 8 hours fasting $(n=9,268)$. Of those, participants with factors that can confound the diagnosis of NAFLD (including excessive alcohol consumption, defined as $>21$ drinks/week in men and $>14$ drinks/week in women [4]; viral hepatitis, defined as positive serum hepatitis B surface antigen and positive serum hepatitis $\mathrm{C}$ antibody; iron overload, defined as transferrin saturation $\geq 50 \%$; or pregnancy) were excluded $(n=1,089)$. The LFS formula includes fasting insulin level, therefore we further excluded participants who were using insulin or other medications for diabetes $(n=315)$. This left 7,864 participants, and once the appropriate exclusion criteria were adopted for each aim (see Additional file 1: Figure S1), we had 5,184 and 5,892 participants included in the analysis of Aim 1 (evaluation of the performance of non-invasive prediction scores of NAFLD in predicting ultrasound-diagnosed NAFLD) and Aim 2 (evaluation of the relationship between non-invasive prediction scores of NAFLD and mortality), respectively.

\section{Definition of NAFLD}

In the original NHANES III between 1988 and 1994, gall bladder ultrasonography video images were recorded using a Toshiba Sonolayer SSA-90A and Toshiba video recorder. Between 2009 and 2010, hepatic steatosis (fatty liver) was assessed by archived video images being rereviewed by three ultrasonography readers (trained by a board-certified radiologist specializing in hepatic imaging), who graded the presence of fat within the hepatic parenchyma.

The following information was recorded on a standard paper collection form: 1) presence of liver-to-kidney contrast; 2) degree of the brightness of the liver parenchyma; 3) presence of deep beam attenuation; 4) presence of echogenic walls in the small intrahepatic vessels, and (5) definition of the gallbladder walls. Finally, an overall primary finding was given based on the presence or absence of each of the five parameters. The liver was graded as having no, mild, moderate, or severe hepatic steatosis. Of the 13,983 participants with hepatic imaging records, 13,856 of them could be graded [7]. Detailed descriptions and procedures have been provided previously $[7,11]$. In the absence of a standard definition, we defined NAFLD as moderate or severe hepatic steatosis, and non-NAFLD as no or mild hepatic steatosis [12]. The overall intra-rater and inter-rater $\kappa$ statistics for reliability of the dichotomized outcomes ("no or mild" and "moderate or severe") were 0.77 (95\% CI 0.73 to 0.82 ) and 0.70 (95\% CI 0.64 to $0.76)$, respectively [11].

\section{Non-invasive markers of NAFLD}

The non-invasive markers of NAFLD were calculated based on the equations reported in the literature [6-9]. In brief, FLI includes body mass index (BMI), $\gamma$-glutamyltranspeptidase, triglycerides, and waist circumference; HSI includes aspartate aminotransferase (AST)/alanine aminotransferase (ALT) ratio, BMI, diabetes, and sex; LAP includes sex, triglycerides, and waist circumference; and LFS includes AST/ALT ratio, diabetes, fasting AST level, fasting insulin level, and MetS. The SteatoTest was not included in the current study, as this test is a commercially one, and the calculation formula is not disclosed. The threshold used in the current study also adopted the cutoff points suggested in the literature: the high/low cutoff points were $\geq 1.257 / \leq-1.413, \geq 30 /<30$, and $\geq 30 /<30$ for LFS, HSI, and FLI, respectively $[6,7,9]$. 


\section{Mortality follow-up}

In NHANES III, cause of death was coded using the International Classification of Diseases. 10th Revision (ICD-10). ICD codes I00 to I78 and E10 to E14 were used to assess cardiovascular and diabetes mortality, respectively, as in our previous studies $[13,14]$. Malignancy and liver mortality were defined by the Underlying Cause of Death (UCOD)_113 20 to 23, 25 to 26, and 43, and UCOD_113 15, 24, and 93 to 95, respectively, as in the literature [15]. The length of follow-up was the time from the study examination date to death or to December 31, 2006, whichever was earlier.

\section{Definition of diabetes, hypertension, and MetS}

Diabetes was defined according to the latest American Diabetes Association (ADA) guideline, which includes fasting glucose $\geq 126 \mathrm{mg} / \mathrm{dl}$, random plasma glucose $\geq$ $200 \mathrm{mg} / \mathrm{dl}$, or $\mathrm{A} 1 \mathrm{C} \geq 6.5$. Patients were considered to have hypertension if they had systolic blood pressure $(\mathrm{SBP}) \geq 140$ or diastolic blood pressure $(\mathrm{DBP}) \geq 90 \mathrm{mmHg}$, or if they were receiving anti-hypertensive drug therapy. MetS was defined according to the joint scientific statement on harmonizing MetS [16], that is, having three or more of the following factors: 1) elevated blood pressure (SBP $\geq 130 \mathrm{mmHg}$ and/or DBP $\geq 85 \mathrm{mmHg}$ and/or being in receipt of anti-hypertension drug therapy); 2) elevated triglycerides $(\geq 150 \mathrm{mg} / \mathrm{dl}(1.7 \mathrm{mmol} / \mathrm{l})$ and/or being in receipt of drug treatment for elevated triglycerides); 3) reduced high-density liproprotein (HDL) cholesterol $(<40 \mathrm{mg} / \mathrm{dl}(1.0 \mathrm{mmol} / \mathrm{l})$ in men and $<50 \mathrm{mg} / \mathrm{dl}$ $(1.3 \mathrm{mmol} / \mathrm{l})$ in women and/or being in receipt of drug treatment for elevated HDL cholesterol); 4) elevated fasting glucose $(\geq 100 \mathrm{mg} / \mathrm{dl}(5.6 \mathrm{mmol} / \mathrm{l})$ and/or being in receipt of treatment for elevated glucose); and 5) large waist circumference $(>102 \mathrm{~cm}$ in men and $>88 \mathrm{~cm}$ in women of European descent). Liver fat percentage was estimated using the equation reported in the same literature as the LFS [7]. The equation includes the same variables as the LFS, but with a different calculation.

\section{Statistical analysis}

To assess model discrimination, we calculated the area under curve (AUC) for the receiver operating characteristic (ROC) for each non-invasive score of NAFLD. The difference between two AUCs was compared using the maximum likelihood estimation method [17] and implemented using ROCKIT [18]. Sensitivity, specificity, positive likelihood ratio (+LR), negative likelihood ratio (-LR), and corresponding 95\% CIs were also calculated. The non-invasive NAFLD measurement with the best performance (in terms of AUC for ROC) was selected and evaluated for its association with mortality.

In the Cox proportional hazard regression model, noninvasive score was modeled as threshold and continuous variables. Using the lower threshold as the reference, the hazard ratio (HR) and 95\% CI for the highest threshold were calculated using the simple and fully adjusted Cox regression models. In the simple model, we adjusted for age and sex. In the full model, we adopted the adjustment model of a recent study related to NAFLD fibrosis and mortality [15], which includes age, sex, race/ethnicity, income, education, diabetes, hypertension, use of lipidlowering medication, smoking, drinking, history of cardiovascular disease (CVD), waist circumference, dietary caffeine intake, HDL cholesterol, triglycerides, transferrin saturation and $C$-reactive protein. $P \leq 0.05$ was considered significant. The proportional hazards assumption was evaluated by including time-dependent covariates in the full regression model; the overall test of proportional hazards was not significant $(P>0.05)$ suggesting that the proportional assumption was valid. To gain additional insight into the potential nonlinearity of the effect of LFS, we examined the Cox regression models using penalized spline. Two degrees of freedom (df) used in the spline because the model had the lowest Akaike's information criterion (AIC) (best fit) when $\mathrm{df}=2$. Sample weights that accounted for the unequal probabilities of selection, oversampling, and non-response were applied in all analyses using the complex sampling module in SPSS (V18.0; SPSS Inc, Chicago, IL, USA) or R software (V2.15.0) [19]. All values presented were weighted to represent the civilian population of the USA.

We also evaluated the ability in risk reclassification using integrated discrimination improvement (IDI) [20] and category-less net reclassification improvement (NRI) [20]. IDI was used to compare the difference in discrimination slopes [21], while category-less NRI was used to compare classifications from two models for changes by outcome for a net calculation of changes in the right direction. Estimated risk of death of different models was calculated using the equation of $1 /(1+\exp (-1 \times \mathrm{XBeta}))$. Analyses were performed using R software (V2.15.0) [19].

\section{Results}

\section{Cross-sectional NAFLD prediction cohort}

Of the 5,184 participants included for the AUC study, $18.4 \%$ (16.5 to $20.4 \%$ ) had NAFLD. The characteristics of this cohort are provided in Table 1 . For NAFLD prediction, LFS was the best performer for predicting NAFLD, with an AUC of $0.771(P<0.001)$, whereas the lowest AUC (0.732) was observed for HSI (Table 2). Using maximum likelihood estimation, the difference between the AUC of LFS and other markers (FLI, LAP, and HSI) was statistically significant (all $P<0.01$ ). Interestingly, the diagnostic accuracy of these markers differed by race/ethnicity (Table 2). The sensitivity and specificity, and the + LR and - LR of the suggested high and low cutoff points for excluding/including NAFLD are provided in Table 3 and 
Table 1 Characteristics of participants in the cross-sectional NAFLD prediction cohort according to NAFLD status ${ }^{a}$

\begin{tabular}{|c|c|c|c|c|c|}
\hline \multirow[t]{2}{*}{ Characteristics } & \multicolumn{2}{|c|}{ No NAFLD $(n=4,117)$} & \multicolumn{2}{|c|}{ NAFLD $(n=1,067)$} & \multirow[t]{2}{*}{$P$ value } \\
\hline & Value & $95 \% \mathrm{Cl}$ & Value & $95 \% \mathrm{Cl}$ & \\
\hline Age, years & 40.42 & 39.59 to 41.25 & 45.32 & 43.97 to 46.67 & $<0.001$ \\
\hline Female sex, \% & 52.6 & 50.4 to 54.7 & 45.6 & 41.7 to 49.5 & 0.005 \\
\hline Race/ethnicity, \% & & & & & $<0.001$ \\
\hline Non-Hispanic white & 74.9 & 71.2 to 78.3 & 73.6 & 69.6 to 77.3 & \\
\hline Non-Hispanic black & 11.6 & 10.1 to 13.4 & 8.7 & 7.3 to 10.3 & \\
\hline Mexican-American & 4.7 & 3.7 to 5.8 & 7.6 & 6.2 to 9.3 & \\
\hline Other & 8.8 & 6.5 to 11.8 & 10.1 & 7.3 to 13.8 & \\
\hline Education & & & & & 0.001 \\
\hline$<$ High school & 5.5 & 4.2 to 7.1 & 8.4 & 6.4 to 11.1 & \\
\hline High school & 14.1 & 12.5 to 16.0 & 18.9 & 15.6 to 22.8 & \\
\hline$>$ High school & 80.4 & 77.7 to 82.8 & 72.6 & 67.8 to 77.0 & \\
\hline Smoking & & & & & $<0.001$ \\
\hline Never & 48.6 & 46.2 to 51.1 & 41.9 & 38.1 to 45.7 & \\
\hline Former & 23.2 & 21.5 to 25.1 & 32.8 & 29.4 to 36.5 & \\
\hline Current & 28.1 & 25.8 to 30.6 & 25.3 & 21.7 to 29.3 & \\
\hline Diabetes, \% & 1.9 & 1.6 to 2.2 & 9.8 & 7.6 to 12.5 & $<0.001$ \\
\hline Hypertension, \% & 16.0 & 14.6 to 17.6 & 33.7 & 29.9 to 37.6 & $<0.001$ \\
\hline Lipid-lowering medication, \% & 2.4 & 1.8 to 3.0 & 5.0 & 3.4 to 7.1 & $<0.001$ \\
\hline History of CVD, \% & 1.8 & 1.3 to 2.3 & 3.9 & 2.8 to 5.4 & 0.002 \\
\hline Metabolic syndrome, \% & 16.9 & 15.3 to 18.7 & 54.8 & 51.2 to 58.5 & $<0.001$ \\
\hline Abdominal obesity, $\%$ & 28.0 & 26.5 to 29.5 & 65.2 & 61.3 to 68.9 & $<0.001$ \\
\hline Hypertriglyceridemia, \% & 22.8 & 20.5 to 25.3 & 54.3 & 50.4 to 58.2 & $<0.001$ \\
\hline Impaired fasting glucose, $\%$ & 20.1 & 18.5 to 21.8 & 42.1 & 38.4 to 46.0 & $<0.001$ \\
\hline Low HDL level, \% & 35.50 & 32.7 to 38.5 & 60.1 & 54.9 to 65.0 & $<0.001$ \\
\hline Body mass index, $\mathrm{kg} / \mathrm{m}^{2}$ & 25.72 & 25.51 to 25.93 & 30.29 & 29.76 to 30.82 & $<0.001$ \\
\hline Waist circumference, cm & 88.82 & 88.35 to 89.3 & 101.75 & 100.36 to 103.13 & $<0.001$ \\
\hline Serum cholesterol, mg/dl & 199.83 & 198.5 to 201.17 & 208.63 & 205.63 to 211.63 & $<0.001$ \\
\hline Serum triglycerides, mg/dl & 117.71 & 114.18 to 121.24 & 192.30 & 183.44 to 201.15 & $<0.001$ \\
\hline Serum HDL cholesterol, mg/dl & $51.04^{\mathrm{b}}$ & 50.15 to 51.93 & $42.91^{d}$ & 41.67 to 44.15 & $<0.001$ \\
\hline Plasma glucose, mg/dl & $94.07^{c}$ & 93.41 to 94.73 & 104.95 & 102.56 to 107.33 & $<0.001$ \\
\hline Serum insulin, $\mu \mathrm{U} / \mathrm{ml}$ & 8.80 & 8.54 to 9.06 & 16.09 & 14.85 to 17.33 & $<0.001$ \\
\hline $\mathrm{SBP}, \mathrm{mmHg}$ & 118.42 & 117.55 to 119.3 & $125.7^{e}$ & 124.7 to 126.7 & $<0.001$ \\
\hline AST, U/L & 19.60 & 19.21 to 20 & 25.24 & 24.31 to 26.17 & $<0.001$ \\
\hline$A L T, U / L$ & 15.70 & 15.03 to 16.36 & 25.68 & 24.27 to 27.09 & $<0.001$ \\
\hline GGT, U/L & 24.42 & 23.69 to 25.15 & 41.61 & 37.77 to 45.44 & $<0.001$ \\
\hline FLI & 34.70 & 33.41 to 35.99 & 67.56 & 64.92 to 70.2 & $<0.001$ \\
\hline HSI & 33.10 & 32.7 to 33.49 & 39.26 & 38.64 to 39.89 & $<0.001$ \\
\hline LFS & -1.92 & -2.03 to to 1.82 & 0.21 & -0.04 to 0.46 & $<0.001$ \\
\hline Liver fat percentage, $\%$ & 2.45 & 2.33 to 2.56 & 5.88 & 5.49 to 6.27 & $<0.001$ \\
\hline LAP & 39.74 & 38.08 to 41.40 & 91.71 & 86.35 to 97.06 & $<0.001$ \\
\hline
\end{tabular}

ALT, alanine aminotransferase: AST, aspartate aminotransferase; CVD, cardiovascular disease; FLI, fatty liver index; GGT, gamma-glutamyl transferase; HDL, high-density lipoprotein; HSI, hepatic steatosis index; LAP, lipid accumulation product; LFS, liver fat score; NAFLD, non-alcoholic fatty liver disease SBP, systolic blood pressure.

${ }^{a}$ No NAFLD: no or mild hepatic steatosis; NAFLD: moderate or severe hepatic steatosis. Numbers of participants: ${ }^{\mathrm{b}} 4108 ;{ }^{\mathrm{c}} 4109 ;{ }^{\mathrm{d}} 1061 ;{ }^{\mathrm{e}} 1049$. 
Table 2 Quality of prediction scores in predicting NAFLD

\begin{tabular}{|c|c|c|c|c|c|c|c|c|c|c|}
\hline \multirow[t]{2}{*}{$\begin{array}{l}\text { NAFLD prediction } \\
\text { scores }\end{array}$} & \multicolumn{2}{|c|}{$\begin{array}{l}\text { All participants } \\
\text { ( } n=5,184 ; 1067 \text { cases) }\end{array}$} & \multicolumn{2}{|c|}{$\begin{array}{l}\text { Non-Hispanic white } \\
\text { ( } n=1,953 ; 376 \text { cases) }\end{array}$} & \multicolumn{2}{|c|}{$\begin{array}{l}\text { Non-Hispanic black } \\
\text { ( } n=1,577 ; 244 \text { cases) }\end{array}$} & \multicolumn{2}{|c|}{$\begin{array}{l}\text { Mexican-American } \\
(n=1,409 ; 401 \text { cases })\end{array}$} & \multicolumn{2}{|c|}{$\begin{array}{l}\text { Others } \\
(n=245 ; 46 \text { cases) }\end{array}$} \\
\hline & AUC & $95 \% \mathrm{Cl}$ & $A \cup C$ & $95 \% \mathrm{Cl}$ & AUC & $95 \% \mathrm{Cl}$ & AUC & $95 \% \mathrm{Cl}$ & AUC & $95 \% \mathrm{Cl}$ \\
\hline LFS & 0.771 & 0.754 to 0.787 & 0.78 & 0.753 to 0.808 & 0.711 & 0.674 to 0.748 & 0.781 & 0.754 to 0.808 & 0.865 & 0.804 to 0.926 \\
\hline FLI & 0.757 & 0.74 to 0.774 & 0.778 & 0.75 to 0.807 & 0.706 & 0.668 to 0.745 & 0.764 & 0.737 to 0.791 & 0.788 & 0.708 to 0.867 \\
\hline LAP & 0.741 & 0.723 to 0.758 & 0.767 & 0.739 to 0.795 & 0.694 & 0.654 to 0.733 & 0.726 & 0.698 to 0.755 & 0.761 & 0.683 to 0.84 \\
\hline HSI & 0.732 & 0.714 to 0.749 & 0.735 & 0.705 to 0.764 & 0.673 & 0.635 to 0.711 & 0.760 & 0.733 to 0.787 & 0.779 & 0.705 to 0.854 \\
\hline
\end{tabular}

AUC, area under the curve; FLI, fatty liver index; HSI, hepatic steatosis index; LAP, lipid accumulation product; LFS, liver fat score; NAFLD, non-alcoholic fatty liver disease.

All $P<0.001$.

Additional file 1: Table S1, respectively. The raw number used to calculate the diagnostic accuracy and the characteristics of true and false positive, and true and false negative (based on the LFS threshold) are provided in Additional file 1: Tables S2 and S3.

\section{Prospective mortality prediction cohort}

Of the several NAFLD non-invasive prediction scores tested, the LFS gave the best performance. As our second aim was to investigate the relationship of the best non-invasive score with outcome, we tested if LFS was associated with mortality. Table 4 shows the characteristics of the participants. During a median follow-up of 14.7 years (range 0.1 to 18.2 years) and $83,830.5$ personyears, 793, 311, 209, 58, and 17 participants died from all, cardiovascular-, malignancy-, diabetes-, and liverrelated causes, respectively. Higher LFS were associated with all causes of mortality tested, except malignancyrelated causes.

The results of Cox regression analysis are shown in Table 5. Participants in the high LFS group had a $60 \%$ higher risk $(\mathrm{HR}=1.6 ; 95 \% \mathrm{CI} 1.01$ to $2.54 ; P=0.048$ in full model) of all-cause mortality than the intermediate LFS group. For cardiovascular mortality, participants in the high LFS group was associated with 2.24-fold (95\% CI 1.03 to 4.88; $P=0.042$ in full model) and 2.3-fold (95\% CI 1.19 to $4.48 ; P=0.015$ in full model) increase in risk of death compared with the low and intermediate LFS groups. For liver mortality, participants in the high LFS group had a 31.25 -fold (95\% CI 3.13 to 333.33; $P=0.004$ in full model) and 30.3 -fold (95\% CI 4 to $250 ; P=0.001$ in full model) increase in risk of death compared with the low and intermediate LFS groups. The Kaplan-Meier survival curves for cardiovascular and liver-related mortality are provided in Figure 1. When LFS was treated as continuous variable, a one-unit increase of LFS was associated with increased mortality of all-cause and cardiovascular-, liver-, and diabetes-related mortality, with HRs of 1.09 (95\% CI 1.01 to $1.19 ; P=0.039$ ), 1.11 (95\% CI 1.03 to 1.19 ; $P=0.006$ ), 1.32 (95\% CI 1.12 to $1.55 ; P=0.001$ ), and 1.21 (95\% CI 1.02 to $1.44 ; P=0.034$ ), respectively (Table 5 ), after full adjustment. Similar results were obtained after further adjustment of the NAFLD fibrosis score (NFS). The relationship between LFS and cardiovascular mortality as examined by penalized regression spline is shown in Figure 2.

Age, sex, hypertension, and diabetes are commonly used in assessing risk of mortality. We therefore evaluated whether addition of LFS (categorical: low/intermediate/ high risk of NAFLD) in a basic clinical model composed of these traditional risk factors could improve the risk prediction. Risk reclassification with IDI showed a modest positive shift to improvement when LFS was added in the basic clinical model (IDI: 0.0131; 95\% CI 0.009 to 0.017;

Table 3 Sensitivity and specificity of exclusion/inclusion cutoff points of LFS, FLI, and HSI in the cross-sectional NAFLD prediction cohort and literature

\begin{tabular}{|c|c|c|c|c|c|c|c|c|c|c|}
\hline \multirow{3}{*}{$\begin{array}{l}\text { NAFLD definition } \\
\text { No to mild versus } \\
\text { intermediate to } \\
\text { severe steatosis) }\end{array}$} & \multirow{3}{*}{$\begin{array}{l}\text { Non-invasive } \\
\text { NAFLD score } \\
\text { LFS }\end{array}$} & \multicolumn{2}{|c|}{ Cutoff point } & \multirow{2}{*}{$\begin{array}{l}\text { SP, \% } \\
96.43\end{array}$} & \multirow{2}{*}{$\begin{array}{l}\mathbf{9 5 \%} \mathrm{Cl}, \% \\
95.82 \text { to } 96.98\end{array}$} & \multirow{2}{*}{$\begin{array}{l}\text { SN, \% } \\
26.34\end{array}$} & \multirow{2}{*}{$\begin{array}{l}\mathbf{9 5 \%} \mathrm{Cl}, \% \\
23.71 \text { to } 29.09\end{array}$} & \multirow{2}{*}{$\begin{array}{l}\text { Reported SP, \% } \\
95\end{array}$} & \multirow{2}{*}{$\begin{array}{l}\text { Reported SN, \% } \\
51\end{array}$} & \multirow{2}{*}{$\begin{array}{l}\text { Ref } \\
{[7]}\end{array}$} \\
\hline & & Inclusion & $\geq 1.257$ & & & & & & & \\
\hline & & Exclusion & $\leq-1.413$ & 67.94 & 66.49 to 69.36 & 73.95 & 71.20 to 76.56 & 52 & 95 & \\
\hline & \multirow[t]{2}{*}{$\mathrm{FLI}$} & Inclusion & $\geq 60$ & 73.60 & 72.22 to 74.94 & 67.48 & 64.58 to 70.29 & 86 & 61 & {$[6]$} \\
\hline & & Exclusion & $<30$ & 48.77 & 47.24 to 50.31 & 84.44 & 82.13 to 86.57 & 64 & 87 & \\
\hline & \multirow[t]{2}{*}{$\mathrm{HSI}$} & Inclusion & $\geq 36$ & 69.35 & 67.91 to 70.75 & 66.26 & 63.33 to 69.10 & 92 & 46 & {$[9]$} \\
\hline & & Exclusion & $<30$ & 29.75 & 28.36 to 31.18 & 91.38 & 89.53 to 92.99 & 40 & 92.50 & \\
\hline
\end{tabular}

AUC, area under the curve; FLI, fatty liver index; HSI, hepatic steatosis index; LAP, lipid accumulation product; LFS, liver fat score; NAFLD, non-alcoholic fatty liver disease; SN, sensitivity; SP, specificity. 
Table 4 Characteristics of participants in the prospective mortality prediction cohort according to different LFS thresholds

\begin{tabular}{|c|c|c|c|c|c|c|c|}
\hline \multirow[t]{2}{*}{ Characteristics } & \multicolumn{2}{|c|}{ Low LFS $(n=3,524)$} & \multicolumn{2}{|c|}{ Intermediate LFS $(n=1,890)$} & \multicolumn{2}{|c|}{ High LFS $(n=478)$} & \multirow[t]{2}{*}{$P$ value } \\
\hline & Value & $95 \% \mathrm{Cl}$ & Value & $95 \% \mathrm{Cl}$ & Value & $95 \% \mathrm{Cl}$ & \\
\hline Age, years & 39.23 & 38.34 to 40.13 & 45.50 & 44.28 to 46.71 & 45.04 & 42.78 to 47.29 & $<0.001$ \\
\hline Sex, female \% & 56.40 & 54.1 to 58.7 & 42.30 & 39.3 to 45.3 & 45.70 & 38.1 to 53.5 & $<0.001$ \\
\hline Race/ethnicity, \% & & & & & & & $<0.001$ \\
\hline Non-Hispanic white & 78.40 & 75.5 to 81.1 & 74.10 & 69.3 to 78.4 & 73.30 & 68.0 to 78.0 & $<0.001$ \\
\hline Non-Hispanic black & 10.30 & 8.9 to 11.8 & 10.50 & 8.8 to 12.5 & 10.90 & 8.6 to 13.7 & $<0.001$ \\
\hline Mexican-American & 4.20 & 3.5 to 5.1 & 6.40 & 5.1 to 8.0 & 7.80 & 5.3 to 11.2 & $<0.001$ \\
\hline Other & 7.10 & 5.4 to 9.3 & 9.00 & 5.9 to 13.4 & 8.00 & 5.6 to 11.5 & $<0.001$ \\
\hline Education, \% & & & & & & & $<0.001$ \\
\hline$<$ High school & 4.10 & 3.2 to 5.2 & 8.20 & 6.0 to 11.0 & 8.10 & 5.5 to 11.7 & $<0.001$ \\
\hline High school & 13.30 & 11.7 to 15.0 & 18.80 & 15.6 to 22.4 & 16.80 & 13.8 to 20.3 & $<0.001$ \\
\hline > High school & 82.60 & 80.3 to 84.7 & 73.10 & 68.4 to 77.3 & 75.10 & 70.1 to 79.6 & $<0.001$ \\
\hline Smoking, \% & & & & & & & $<0.001$ \\
\hline Never & 49.20 & 46.2 to 52.3 & 41.00 & 37.3 to 44.8 & 47.90 & 40.3 to 55.5 & $<0.001$ \\
\hline Former & 21.60 & 19.6 to 23.7 & 33.20 & 29.5 to 37.1 & 32.30 & 25.9 to 39.5 & $<0.001$ \\
\hline Current & 29.20 & 26.4 to 32.2 & 25.80 & 23.1 to 28.6 & 19.80 & 14.1 to 27.1 & $<0.001$ \\
\hline Diabetes, \% & 0.20 & 0.1 to 0.3 & 5.70 & 4.6 to 7.0 & 26.20 & 21.3 to 31.7 & $<0.001$ \\
\hline Hypertension, \% & 10.00 & 8.7 to 11.4 & 32.70 & 29.6 to 36.0 & 45.40 & 39.6 to 51.3 & $<0.001$ \\
\hline Lipid-lowering medication, \% & 1.10 & .7 to 1.8 & 5.40 & 4.1 to 7.1 & 7.20 & 4.4 to 11.6 & $<0.001$ \\
\hline History of CVD, \% & 1.30 & .9 to 1.9 & 3.40 & 2.4 to 4.8 & 5.10 & 3.1 to 8.2 & $<0.001$ \\
\hline Poverty income ratio & 3.25 & 3.09 to 3.4 & 2.98 & 2.76 to 3.2 & 2.83 & 2.54 to 3.11 & 0.003 \\
\hline Waist circumference, cm & 85.22 & 84.74 to 85.71 & 100.22 & 99.52 to 100.92 & 112.22 & 110.51 to 113.93 & $<0.001$ \\
\hline Dietary caffeine intake, mg & 272.81 & 251.75 to 293.87 & 276.43 & 234.41 to 318.45 & 216.06 & 189.12 to 243 & 0.222 \\
\hline Serum cholesterol, mg/dl & 196.06 & 194.01 to 198.11 & 213.02 & 209.91 to 216.14 & 218.33 & 211.56 to 225.1 & $<0.001$ \\
\hline Serum HDL cholesterol, mg/dl & 53.38 & 52.51 to 54.25 & 43.81 & 42.74 to 44.89 & 39.06 & 37.89 to 40.23 & $<0.001$ \\
\hline Serum transferrin saturation, \% & 27.78 & 27.25 to 28.31 & 26.25 & 25.55 to 26.95 & 25.54 & 23.88 to 27.2 & $<0.001$ \\
\hline Serum C-reactive protein, $\mathrm{mg} / \mathrm{dl}$ & 0.33 & 0.31 to 0.35 & 0.45 & 0.42 to 0.49 & 0.58 & 0.5 to 0.66 & $<0.001$ \\
\hline Drinks/week ${ }^{\mathrm{a}}$ & 2.68 & 2.45 to 2.9 & 2.32 & 2.01 to 2.64 & 1.62 & 1.11 to 2.13 & $<0.001$ \\
\hline LFS & -2.53 & -2.58 to -2.48 & -0.41 & -0.46 to -0.36 & 3.20 & 2.83 to 3.57 & $<0.001$ \\
\hline \multicolumn{8}{|l|}{ Mortality } \\
\hline All causes & 8.90 & 7.7 to 10.2 & 13.60 & 11.4 to 16.2 & 17.70 & 12.9 to 23.9 & $<0.001$ \\
\hline CVD-related & 2.80 & 2.3 to 3.5 & 5.10 & 4.0 to 6.4 & 9.00 & 5.4 to 14.8 & $<0.001$ \\
\hline Liver-related & 0.10 & 0.0 to 0.3 & 0.10 & 0.0 to 0.2 & 1.20 & 0.3 to 5.2 & 0.069 \\
\hline Diabetes-related & 0.20 & 0.1 to 0.5 & 0.90 & 0.6 to 1.5 & 3.90 & 2.0 to 7.4 & $<0.001$ \\
\hline Malignancy-related & 2.80 & 2.1 to 3.8 & 3.60 & 2.5 to 5.2 & 2.00 & 1.0 to 4.2 & 0.698 \\
\hline
\end{tabular}

CVD, cardiovascular disease; LFS, liver fat score; NAFLD, non-alcoholic fatty liver disease.

${ }^{a} A$ drink was defined as a 12-oz beer, a 4-oz glass of wine, or 1-oz of liquor (spirits).

$P<0.001)$. Similar result was observed using categoryless NRI (NRI: $0.133 ; 95 \%$ CI 0.054 to $0.211 ; P<0.001$ ).

\section{Discussion}

Using a large, nationally representative cohort with more than 10 years of follow-up and ultrasonographic data, we have demonstrated that LFS is the best prediction score for ultrasonography-diagnosed NAFLD, and can predict mortality, including cardiovascular- and liverrelated mortality.

It is important to find an easy and cost-effective way to screen for NAFLD. Of the several non-invasive scores we tested, LFS showed the best performance in identifying ultrasonography-diagnosed NAFLD. Notably, LFS 
Table 5 Association between LFS and mortality

\begin{tabular}{|c|c|c|c|c|c|c|c|c|c|}
\hline \multirow[t]{2}{*}{ Mortality from: } & \multicolumn{3}{|c|}{ Simple model $^{a}$} & \multicolumn{3}{|c|}{ Full model ${ }^{\mathbf{b}}$} & \multicolumn{3}{|c|}{ Full model + NFS adjustment ${ }^{b}$} \\
\hline & $\mathrm{HR}$ & $95 \% \mathrm{Cl}$ & $P$ value & HR & $95 \% \mathrm{Cl}$ & $P$ value & $\mathrm{HR}$ & $95 \% \mathrm{Cl}$ & $P$ value \\
\hline \multicolumn{10}{|l|}{ All causes } \\
\hline Low (ref) versus high LFS & 1.51 & 0.99 to 2.3 & 0.056 & 1.59 & 0.93 to 2.72 & 0.087 & 1.65 & 0.96 to 2.85 & 0.07 \\
\hline Int (ref) versus high LFS & 1.52 & 1.03 to 2.23 & 0.034 & 1.60 & 1.01 to 2.54 & 0.048 & 1.61 & 1 to 2.58 & 0.049 \\
\hline LFS (continuous) & 1.07 & 0.99 to 1.14 & 0.082 & 1.09 & 1.01 to 1.19 & 0.039 & 1.1 & 1.024 to 1.19 & 0.011 \\
\hline \multicolumn{10}{|l|}{ CVD } \\
\hline Low (ref) versus high LFS & 2.29 & 1.24 to 4.26 & 0.009 & 2.24 & 1.03 to 4.88 & 0.042 & 2.30 & 1.02 to 5.19 & 0.046 \\
\hline Int (ref) versus high LFS & 2.20 & 1.18 to 4.12 & 0.015 & 2.30 & 1.19 to 4.48 & 0.015 & 2.32 & 1.18 to 4.55 & 0.015 \\
\hline LFS (continuous) & 1.11 & 1.04 to 1.19 & 0.002 & 1.11 & 1.03 to 1.19 & 0.006 & 1.13 & 1.05 to 1.21 & $<0.001$ \\
\hline \multicolumn{10}{|l|}{ Liver disease } \\
\hline Low (ref) versus high LFS & 9.80 & 2.01 to 47.62 & 0.006 & 31.25 & 3.13 to 333.33 & 0.004 & 46.45 & 5.13 to 420.51 & $<0.001$ \\
\hline Int (ref) versus high LFS & 17.24 & 2.6 to 111.11 & 0.004 & 30.30 & $4-250$ & 0.001 & 32.99 & 4.57 to 237.95 & $<0.001$ \\
\hline LFS (continuous) & 1.25 & 1.14 to 1.36 & $<0.001$ & 1.32 & 1.12 to 1.55 & 0.001 & 1.3 & 1.12 to 1.51 & $<0.001$ \\
\hline \multicolumn{10}{|l|}{ Diabetes } \\
\hline Low (ref) versus high LFS & 15.87 & 6.02 to 41.67 & $<0.001$ & 2.87 & 0.65 to 12.66 & 0.161 & 3.13 & 0.67 to 14.56 & 0.143 \\
\hline Int (ref) versus high LFS & 5.24 & 2.24 to 12.2 & $<0.001$ & 1.98 & 0.71 to 5.52 & 0.185 & 2.04 & 0.72 to 5.79 & 0.176 \\
\hline LFS (continuous) & 1.37 & 1.27 to 1.48 & $<0.001$ & 1.21 & 1.02 to 1.44 & 0.034 & 1.21 & 1.03 to 1.42 & 0.024 \\
\hline \multicolumn{10}{|l|}{ Malignancy } \\
\hline Low (ref) versus high LFS & 0.55 & 0.25 to 1.24 & 0.147 & 0.75 & 0.27 to 2.11 & 0.578 & 0.75 & 0.27 to 2.09 & 0.576 \\
\hline Int (ref) versus high LFS & 0.65 & 0.29 to 1.44 & 0.281 & 0.76 & 0.32 to 1.79 & 0.519 & 0.76 & 0.32 to 1.79 & 0.518 \\
\hline LFS (continuous) & 0.92 & 0.86 to 0.99 & 0.026 & 0.97 & 0.87 to 1.07 & 0.521 & 0.95 & 0.8 to 1.12 & 0.495 \\
\hline
\end{tabular}

CVD, cardiovascular disease; Int, intermediate; LFS, liver fat score.

aSimple model: adjusted for age and sex.

${ }^{b}$ Full model: further adjusted for race/ethnicity, education, income, diabetes, hypertension, history of CVD, lipid-lowering medication, smoking status, waist circumference, alcohol consumption, caffeine consumption, total cholesterol, high-density lipoprotein cholesterol, transferrin saturation, and C-reactive protein; Because of smaller numbers of liver- and diabetes-related deaths, we did not adjust for education, income, history of CVD, lipid-lowering medication, and C-reactive protein in the full model.

was derived using ${ }^{1} \mathrm{H}$-MRS-diagnosed NAFLD, whereas FLI, HSI, and LAP were derived from ultrasonographydiagnosed NAFLD. For those scores derived from ultrasonography, the definitions of the NAFLD were also somewhat different (see Additional file 1: Table S4). Ultrasonography is a semi-quantitative imaging technique, and the definitions of NAFLD differed between studies. By contrast, ${ }^{1} \mathrm{H}$-MRS is by far the most sensitive and quantitative imaging tool in identifying hepatic steatosis. This could be the reason why the non-invasive score (LFS) derived from ${ }^{1} \mathrm{H}$-MRS performed better and more robustly in identifying cases in the current study. As there is no standard definition of ultrasonography-diagnosed NAFLD for good measurement, we used three additional definitions to test the performance of different noninvasive indices (see Additional file 1: Table S5), and the LFS still came out best. We evaluated whether combining all prediction scores (combined score) could improve the NAFLD prediction. The AUC of the combined score increased to 0.782 ( $95 \%$ CI 0.766 to 0.798 ), suggesting that there are unique NAFLD predicting components being captured in different prediction scores.
Interestingly, there was a difference in the diagnostic accuracy of the different non-invasive scores, with the lowest diagnostic accuracy being observed in black patients for all tested scores, suggesting that the clinical risk factors of NAFLD could be ethnicity-specific and particularly different in black populations. Like other disease predictions [22], deriving an ethnicity- or populationspecific prediction model may be required to achieve a high accuracy of NAFLD prediction. Notably, although there was an observed difference in the diagnostic accuracy of LFS for NAFLD, no significant interaction $(P>0.05)$ between LFS and race/ethnicity on mortality was observed, therefore, no subgroup analysis was performed in the subsequent analyses.

LFS was calculated based on the AST/ALT ratio, diabetes, fasting AST level, fasting insulin level, and MetS. Given that diabetes and MetS are known to be associated with mortality, the association between LFS and mortality could be attributable to these factors. However, the components of MetS were adjusted for in the full model, suggesting that the association of LFS with mortality may be independent of these factors. A number of 


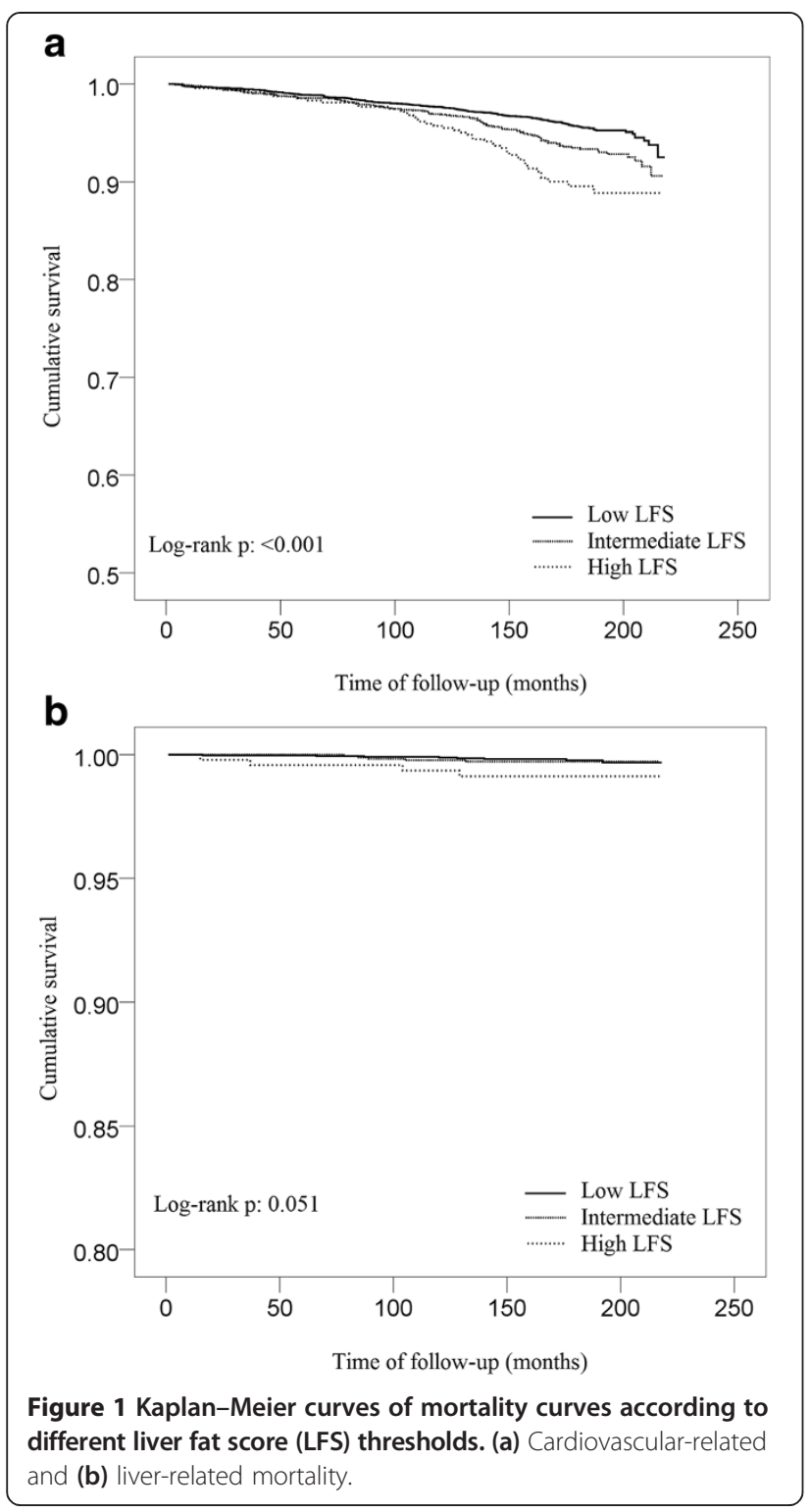

different organizations use different recommended waist circumference thresholds for abdominal obesity in defining MetS. In addition to the threshold suggested by ATPIII [16], we also used the population-specific threshold suggested by the International Diabetes Federation, and the findings remained unchanged (data not shown).

In the literature on FLI, LFS, and HSI, various high and low cutoff points have been proposed to include and exclude NAFLD $[6,7,9]$. The high cutoff point should have a high specificity and + LR, while the low cutoff point should have a high sensitivity and low - LR. In general, the diagnostic performance of the defined cutoff points of the NAFLD prediction scores was not the same as that originally reported in the literature because the sample populations were different (Table 3 ). The high cutoff point of LFS had a slightly higher specificity $(96.4 \%)$ in the current study than the figure (95\%) reported in the literature, meaning that study participants with a high LFS were very likely to have a higher risk of mortality.

Two previous studies validated the non-invasive prediction scores in adults [23,24]. Koehler et al. validated FLI and LAP in 2,652 participants in the Rotterdam Study. FLI and LAP had an AUC of 0.813 and 0.786, respectively, in predicting ultrasonography-diagnosed NAFLD. FLI had a higher AUC in the Rotterdam Study than in the current study. Interestingly, the Rotterdam Study used the scoring protocol of Hamaguchi et al. [25], and the one used by NHANES was an algorithm derived based on that same publication. The Rotterdam Study was a populationbased cohort study of elderly inhabitants of a district of Rotterdam, whereas NHANES was a nationally representative population-based study with participants of different races/ethnicities and age. LFS was previously validated in a study of 40 non-diabetic patients with biopsy-proven NAFLD and 85 healthy controls [23], which showed that LFS had an AUC of 0.86. Although the AUC from different validation studies cannot be compared directly, our study is in agreement with previous

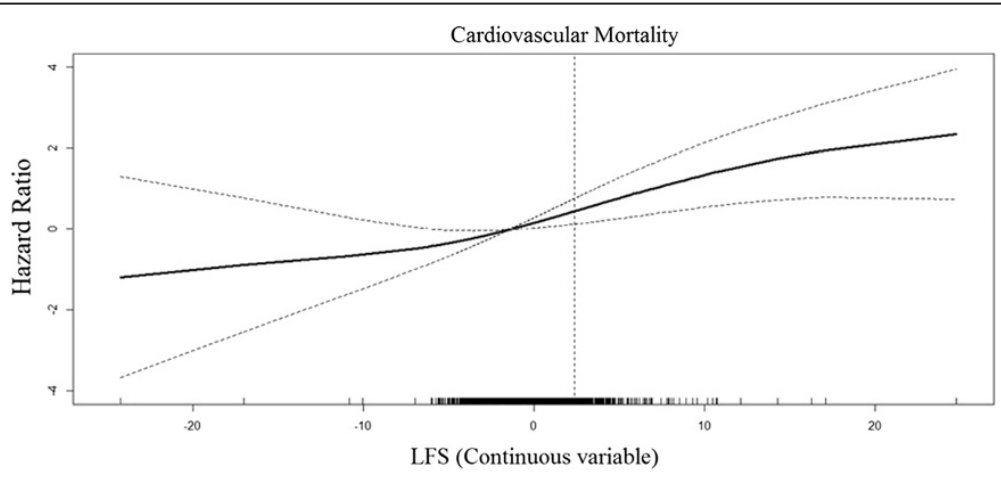

Median was used as the reference. The vertical dash line indicates the high LFS cutoff point.

Figure 2 Association between liver fat score (LFS) and cardiovascular mortality via penalized regression splines. 
validation studies showing that LFS had the highest AUC, followed by FLI and LAP. Although no validation study has been performed for HSI, our study suggested that HSI is better than LAP as a predictor of NAFLD.

Although identifying people with NAFLD is important, identifying people with adverse clinical outcome is even more important, as NAFLD consists of a wide spectrum of conditions, ranging from simple steatosis to cirrhosis with varying prognosis. In concordance with previous NHANES reports $[12,15]$, our study did not reveal any significant association between ultrasonographydiagnosed NAFLD and mortality (data not shown). This finding is intriguing. Ultrasonography-diagnosed NAFLD is not associated with mortality, whereas LFS, a marker of NAFLD, is associated with mortality. This could be due to the reason mentioned earlier, namely, that LFS was derived using the sensitive and quantitative imaging tool ${ }^{1} \mathrm{H}$-MRS, whereas other NAFLD prediction scores were derived using a less sensitive semi-quantitative ultrasonography. In fact, we found no association of other markers of NAFLD with mortality (se Additional file 1: Table S5), further suggesting that ${ }^{1} \mathrm{H}$-MRS-derived LFS may be more superior in identifying NAFLD and predicting clinical outcome.

We then investigated which individual component of the LFS was associated with CVD mortality in the multivariable model, and found the only significant association observed Qa with fasting serum insulin (estimate of $1.02 ; 95 \%$ CI 1.01 to $1.03 ; P<0.001$ ), suggesting that fasting serum insulin may be the main driver for the observed association.

Notably, high LFS is also associated with low transferrin saturation (Table 4). In our previous study, we showed that low transferrin saturation was robustly associated with pre-diabetes [26]. These observations suggested that elevated insulin resistance might be the key factor leading to mortality in people with NAFLD, which may also explain why LFS can predict mortality whereas ultrasonography-diagnosed NAFLD cannot. Another possibility is that high LFS indicates the presence of other NAFLD-related conditions, such as NASH and fibrosis. In participants with NAFLD, high LFS is associated with high NFS (data not shown), which is a prediction score of NAFLD fibrosis [27], although NFS does not predict NAFLD (data not shown) nor is it significantly associated with mortality in the general population (see Additional file 1: Table S5). However, further adjustment of NFS revealed that the effect of LFS is independent of NFS (Table 5), suggesting that the association between LFS and mortality may be independent of NAFLD fibrosis. Future study is required to confirm our observations and to examine the underlying mechanisms.

Age, sex, and presence of diabetes or hypertension are simple risk factors that are commonly used by clinicians to evaluate mortality risk. We showed that LFS has an independent role in predicting mortality and improved risk reclassification. Similarly, although the Framingham Risk Score (FRS) was not intended for use in mortality prediction, we found that the associations of LFS with cardiometabolic disease related mortality were independent of FRS (see Additional file 1: Table S6). These findings suggest that abnormal liver function may play a role in mortality determination, independently of traditional risk factors.

Our study has several strengths. The study population is large, multiethnic, nationally representative, and wellcharacterized, with data on ultrasonography-diagnosed NAFLD, multiple risk factors, and potential confounders. The long follow-up and the large number of events provided ample statistical power. The wide range of collected data from NHANES III allowed construction of four different non-invasive prediction scores simultaneously, so that they could be compared in parallel and with different definitions of NAFLD.

Nevertheless, there are limitations. The major limitation of the current study is the use of ultrasonographydiagnosed NAFLD, which can lead to misclassification error. In the absence of a standard definition, we defined NAFLD as presence of moderate or severe hepatic steatosis, while non-NAFLD was defined as presence of no or mild hepatic steatosis, as in previous study. The case definition, especially when mild hepatic steatosis was defined as non-NAFLD, could be a potential source of bias. This classification could have led to underestimation of the NAFLD prevalence in the current study, which was reported to be $20 \%$ to $33 \%$ in the general population [28], although the lower prevalence observed could also be due to the lower prevalence of obesity in the current study [29]. It is acknowledged that ultrasonography has limited sensitivity and specificity in diagnosing NAFLD, especially when less than $33 \%$ of the liver parenchyma is infiltrated by fat $[30,31]$ and in the presence of liver cirrhosis that may lead to decreased hepatic steatosis. To confirm our findings, we defined NAFLD in different ways and still found that LFS was the best marker of NAFLD (Table 4; see Additional file 1: Table S7), and participants with mild hepatic steatosis also did not have increased mortality compared with those without hepatic steatosis (see Additional file 1: Table S8).

Although liver biopsy is considered the gold standard in diagnosing NAFLD, it is not justifiable to perform liver biopsy in large numbers of asymptomatic individuals, therefore ultrasonography is still considered an acceptable first-line screening procedure for NAFLD in clinical practice [32]. However, ultrasonography cannot distinguish between NASH, fibrosis or cirrhosis.

The prediction score named the SteatoTest, was not included in the current study; whether it is superior to LFS or otherwise requires further study. 
In the Cox regression analysis, there were too few cases of liver-related mortality, which led to unreliable estimates, and could also be a potential source of bias, therefore cautious interpretation is required.

\section{Conclusion}

In conclusion, we found that ${ }^{1} \mathrm{H}$-MRS derived NAFLD prediction score LFS was the most robust non-invasive score identifying NAFLD in this US population and predicted mortality. NAFLD is highly prevalent, and can be associated with morbidity and mortality if left unidentified. Our findings suggest that LFS may be a promising tool for large-scale NAFLD screening. If confirmed in future studies, LFS may be a useful marker for largescale NAFLD screening and prediction of long-term clinical outcomes.

\section{Additional file}

Additional file 1: Additional file contains supplementary Tables S1 to S8 and supplementary Figure S1.

\section{Competing interests}

The authors declare that they have no competing interests.

\section{Authors' contributions}

$\mathrm{CLC}$ was responsible for study conception and design; data generation and analysis; and manuscript drafting. CLC, KSLL, ICKW, BMYC were responsible for data interpretation. All authors reviewed, commented upon, and approved the final submission.

\section{Authors' information}

$\mathrm{CLC}$ is the article guarantor.

\section{Acknowledgements}

BMYC received support from the Sun Chieh Yeh Heart Foundation and CLC was supported by the Faculty Development Fund (Li Ka Shing Faculty of Medicine, University of Hong Kong), and the PDF/RAP Scheme (University of Hong Kong).

\section{Author details \\ 'Department of Medicine, Li Ka Shing Faculty of Medicine, The University of Hong Kong, Pokfulam, Hong Kong. ${ }^{2}$ Research Centre of Heart, Brain, Hormone and Healthy Aging, Li Ka Shing Faculty of Medicine, The University of Hong Kong, Pokfulam, Hong Kong. ${ }^{3}$ Department of Pharmacology and Pharmacy, Li Ka Shing Faculty of Medicine, The University of Hong Kong, Pokfulam, Hong Kong. ${ }^{4}$ Centre for Genomic Sciences, Li Ka Shing Faculty of Medicine, The University of Hong Kong, Pokfulam, Hong Kong. ${ }^{5}$ The State Key Laboratory of Pharmaceutical Biotechnology, Li Ka Shing Faculty of Medicine, The University of Hong Kong, Pokfulam, Hong Kong.}

Received: 12 June 2014 Accepted: 15 August 2014

Published online: 10 September 2014

\section{References}

1. Targher G, Byrne CD: Clinical Review: Nonalcoholic fatty liver disease: a novel cardiometabolic risk factor for type 2 diabetes and its complications. J Clin Endocrinol Metab 2013, 98:483-495.

2. Targher G, Day CP, Bonora E: Risk of cardiovascular disease in patients with nonalcoholic fatty liver disease. N Engl I Med 2010, 363:1341-1350.

3. Anstee QM, Targher G, Day CP: Progression of NAFLD to diabetes mellitus, cardiovascular disease or cirrhosis. Nat Rev Gastroenterol Hepatol 2013, 10:330-344
4. Chalasani N, Younossi Z, Lavine JE, Diehl AM, Brunt EM, Cusi K, Charlton M, Sanyal AJ: The diagnosis and management of non-alcoholic fatty liver disease: practice Guideline by the American Association for the Study of Liver Diseases, American College of Gastroenterology, and the American Gastroenterological Association. Hepatology 2012, 55:2005-2023.

5. Poynard T, Ratziu V, Naveau S, Thabut D, Charlotte F, Messous D, Capron D, Abella A, Massard J, Ngo Y, Munteanu M, Mercadier A, Manns M, Albrecht J: The diagnostic value of biomarkers (SteatoTest) for the prediction of liver steatosis. Comp Hepatol 2005, 4:10.

6. Bedogni G, Bellentani S, Miglioli L, Masutti F, Passalacqua M, Castiglione A, Tiribelli C: The Fatty Liver Index: a simple and accurate predictor of hepatic steatosis in the general population. BMC Gastroenterol 2006, 6:33.

7. Kotronen A, Peltonen M, Hakkarainen A, Sevastianova K, Bergholm R, Johansson LM, Lundbom N, Rissanen A, Ridderstråle M, Groop L, Orho-Melander M, Yki-Järvinen H: Prediction of non-alcoholic fatty liver disease and liver fat using metabolic and genetic factors. Gastroenterology 2009, 137:865-872.

8. Bedogni G, Kahn HS, Bellentani S, Tiribelli C: A simple index of lipid overaccumulation is a good marker of liver steatosis. BMC Gastroenterol 2010, 10:98.

9. Lee JH, Kim D, Kim HJ, Lee CH, Yang Jl, Kim W, Kim YJ, Yoon JH, Cho SH, Sung MW, Lee HS: Hepatic steatosis index: a simple screening tool reflecting nonalcoholic fatty liver disease. Dig Liver Dis 2010, 42:503-508.

10. Centers for Disease Control and Prevention (CDC): National Center for Health Statistics (NCHS). National Health and Nutrition Examination Survey Data. Hyattsville, MD: U.S. Department of Health and Human Services, Centers for Disease Control and Prevention [1988-1994]; 1996. Available from http:// www.cdc.gov/nchs/nhanes/nh3data.htm.

11. Hepatic Steatosis Ultrasound Images Assessment Procedures Manual: Hepatic Steatosis Ultrasound Images Assessment Procedures Manual. Available from http://www.cdc.gov/nchs/data/nhanes/nhanes3/ hepatic_steatosis_ultrasound_procedures_manual.pdf. Available from http:// www.cdc.gov/nchs/nhanes/nhanes3/HGUHS.htm, accessed 25/01/2014.

12. Lazo M, Hernaez R, Bonekamp S, Kamel IR, Brancati FL, Guallar E, Clark JM: Non-alcoholic fatty liver disease and mortality among US adults: prospective cohort study. BMJ 2011, 343:d6891.

13. Cheung CL, Lam KS, Cheung BM: Serum beta-2 microglobulin concentration predicts cardiovascular and all-cause mortality. Int J Cardiol 2013, 168:4811-4813.

14. Cheung CL, Lam KS, Cheung BM: Serum beta-2 microglobulin predicts mortality in people with diabetes. Eur J Endocrinol 2013, 169:1-7.

15. Kim D, Kim WR, Kim HJ, Therneau TM: Association between noninvasive fibrosis markers and mortality among adults with nonalcoholic fatty liver disease in the United States. Hepatology 2013, 57:1357-1365.

16. Alberti KG, Eckel RH, Grundy SM, Zimmet PZ, Cleeman JI, Donato KA, Fruchart JC, James WP, Loria CM, Smith SC Jr, International Diabetes Federation Task Force on Epidemiology and Prevention; Hational Heart, Lung, and Blood Institute; American Heart Association; World Heart Federation; International Atherosclerosis Society; International Association for the Study of Obesity: Harmonizing the metabolic syndrome: a joint interim statement of the International Diabetes Federation Task Force on Epidemiology and Prevention; National Heart, Lung, and Blood Institute; American Heart Association; World Heart Federation; International Atherosclerosis Society; and International Association for the Study of Obesity. Circulation 2009, 120:1640-1645.

17. Metz CE, Herman BA, Shen JH: Maximum likelihood estimation of receiver operating characteristic (ROC) curves from continuously-distributed data. Stat Med 1998, 17:1033-1053.

18. Metz CE. ROCKIT. [http://metz-roc.uchicago.edu/MetzROC/software]

19. R project. [www.R-project.org]

20. Pencina MJ, D'Agostino RB Sr, D'Agostino RB Jr, Vasan RS: Evaluating the added predictive ability of a new marker: from area under the ROC curve to reclassification and beyond. Stat Med 2008, 27:157-172. discussion 207-112.

21. Steyerberg EW, Vickers AJ, Cook NR, Gerds T, Gonen M, Obuchowski N, Pencina MJ, Kattan MW: Assessing the performance of prediction models: a framework for traditional and novel measures. Epidemiology 2010, 21:128-138.

22. Cheung EY, Bow CH, Cheung CL, Soong C, Yeung S, Loong C, Kung A: Discriminative value of FRAX for fracture prediction in a cohort of Chinese postmenopausal women. Osteoporos Int 2012, 23:871-878. 
23. Musso G, Gambino R, Durazzo M, Cassader M: Noninvasive assessment of liver disease severity with liver fat score and CK-18 in NAFLD: prognostic value of liver fat equation goes beyond hepatic fat estimation. Hepatology 2010, 51:715-717.

24. Koehler EM, Schouten JN, Hansen BE, Hofman A, Stricker BH, Janssen HL: External validation of the fatty liver index for identifying nonalcoholic fatty liver disease in a population-based study. Clin Gastroenterol Hepatol 2013, 11:1201-1204.

25. Hamaguchi M, Kojima T, Itoh Y, Harano Y, Fujii K, Nakajima T, Kato T, Takeda N, Okuda J, Ida K, Kawahito Y, Yoshikawa T, Okanoue T: The severity of ultrasonographic findings in nonalcoholic fatty liver disease reflects the metabolic syndrome and visceral fat accumulation. Am J Gastroenterol 2007, 102:2708-2715.

26. Cheung CL, Cheung TT, Lam KS, Cheung BM: High ferritin and low transferrin saturation are associated with pre-diabetes among a national representative sample of U.S. adults. Clin Nutr 2013, 32:1055-1060.

27. Angulo P, Hui JM, Marchesini G, Bugianesi E, George J, Farrell GC, Enders F, Saksena S, Burt AD, Bida JP, Lindor K, Sanderson SO, Lenzi M, Adams LA, Kench J, Therneau TM, Day CP: The NAFLD fibrosis score: a noninvasive system that identifies liver fibrosis in patients with NAFLD. Hepatology 2007, 45:846-854.

28. Browning JD, Szczepaniak LS, Dobbins R, Nuremberg P, Horton JD, Cohen JC, Grundy SM, Hobbs HH: Prevalence of hepatic steatosis in an urban population in the United States: impact of ethnicity. Hepatology 2004, 40:1387-1395.

29. Lazo M, Hernaez R, Eberhardt MS, Bonekamp S, Kamel I, Guallar E, Koteish A, Brancati FL, Clark JM: Prevalence of nonalcoholic fatty liver disease in the United States: the Third National Health and Nutrition Examination Survey, 1988-1994. Am J Epidemiol 2013, 178:38-45.

30. Ryan CK, Johnson LA, Germin BI, Marcos A: One hundred consecutive hepatic biopsies in the workup of living donors for right lobe liver transplantation. Liver Transp/ 2002, 8:1114-1122.

31. Saadeh S, Younossi ZM, Remer EM, Gramlich T, Ong JP, Hurley M, Mullen $K D$, Cooper JN, Sheridan MJ: The utility of radiological imaging in nonalcoholic fatty liver disease. Gastroenterology 2002, 123:745-750.

32. Ratziu V, Bellentani S, Cortez-Pinto H, Day C, Marchesini G: A position statement on NAFLD/NASH based on the EASL 2009 special conference. J Hepatol 2010, 53:372-384.

doi:10.1186/s12916-014-0154-x

Cite this article as: Cheung et al:: Non-invasive score identifies ultrasonography-diagnosed non-alcoholic fatty liver disease and predicts mortality in the USA. BMC Medicine 2014 12:154.

\section{Submit your next manuscript to BioMed Central and take full advantage of:}

- Convenient online submission

- Thorough peer review

- No space constraints or color figure charges

- Immediate publication on acceptance

- Inclusion in PubMed, CAS, Scopus and Google Scholar

- Research which is freely available for redistribution 